\title{
Estrogen Receptor, Progesterone Receptor, and Her-2/neu Oncogene Expression in Breast Cancers Among Bangladeshi Women
}

\author{
MG MOSTAFA ${ }^{\mathrm{a}}$, MT LARSEN ${ }^{\mathrm{b}}, \mathrm{RR}_{\text {LOVE }}^{\mathrm{b}}$
}

\begin{abstract}
Summary:
Two-thirds of all women who develop breast cancer each year live in Asia. In many countries, including Bangladesh, there are few data on the pathological characteristics of breast tumours. The objectives of this study were a) to describe the estrogen receptor $(E R)$, progesterone receptor $(P R)$, and the expression of Her-2/neu oncogene expression status in a large series of breast cancers occurring in Bangladeshi women and b) to correlate these findings with the patients' age at diagnosis, tumour histological grade, and presence of axillary lymph node metastatic disease.
\end{abstract}

Method: One thousand forty two cases were evaluated in a referral practice. Tumour sections were stained immunohistochemically using Dako 1D5 (ER) and Dako 636 (PR) and semiquantitatively scored for ER and PR expression. Three hundred thirty five of these cases were also stained using Dako c-erb2 oncoprotein and scored for Her-2/neu over-expression.

\section{Introduction:}

The causes of cancer and time of its appearance varying among populations and thus it may be expected that the biological characteristics of tumours among populations would differ. However, for breast cancer, with the exception of greater frequencies of inherited cancer susceptibility genes such as BRCA 1 and 2, there has been little evidence of different causes among populations and generally when population comparisons have been carefully made. The most commonly described biological characteristics of tumours - the histological types and grades, and hormonal receptor expression have been similar across populations ${ }^{1}$. Nevertheless, in some

a. Mohammad Golam Mostafa, M.B.B.S., M. Phil (pathology), The National Institute for Cancer Research Hospital Dhaka and Consultant Anwara Diagnostic Center, Dhaka (MGM)

b. Michael T. Larsen, The College of Medicine, The Ohio State University, Columbus, Ohio, U.S.A. (MTL and RRL), Richard R. Love, M.D, The College of Medicine, The Ohio State University, Columbus, Ohio, U.S.A. (MTL and RRL).

Address of Correspondence: Mohammad Golam Mostafa, email: dr.mohammadgolammostafa@gmail.com

Received: 20 June, 2009

Accepted: 26 May, 2010
Results: Estrogen Receptor expression was positive in $69.0 \%, P R$ expression was positive in $72.3 \%$, and Her-2/ neu was over-expressed (IHC score $3+$ ) in $28.4 \%$ of the cases. Her-2/neu over-expression did not consistently correlate with $E R$ and $P R$ expression. $E R$ and $P R$ expression were inversely associated with tumour histological grade. Cases with axillary lymph node metastases had higher rates of $E R$ and PR expression. No significant association was observed with patient's age.

Conclusion: Estrogen Receptor, PR, and Her-2/neu expression frequencies and prognostic factor associations in Bangladeshi women with breast cancer referred for tumour marker testing are very similar to those reported in Western countries. These findings have important implications for ensuring optimal testing capacity for all patients with these tumours, to allow for appropriate choices of treatment.

(J Bangladesh Coll Phys Surg 2010; 28: 157-162)

countries, including Bangladesh, there has been a widespread impression that the frequencies of expression of hormonal receptor proteins in breast tumours are significantly lower than those seen in western women. Such impressions have led to reluctance to even assess patients' tumours for these proteins because "the tumours are always negative”. In these circumstances, women are not offered the possibility of hormonal treatment, from which benefits are only expected in the face of expression of hormonal receptor proteins in the tumours ${ }^{2}$. Her-2/ neu over-expression in tumours has been associated with resistance to one form of hormonal therapy that is with tamoxifen $^{3}$.

In these contexts the objectives of the current study were, in a large series of cases of breast cancer among Bangladeshi women: a) to ascertain the estrogen receptor (ER) status, the progesterone receptor (PR) status, and the over-expression of Her-2/neu oncogene; and b) to evaluate correlations of these results with each other and with patients' ages at diagnosis, tumour histological grading, and evidence of axillary metastatic 
lymph node involvement in those patients with clinical stages I-III breast-operated disease.

\section{Materials and Methods:}

A retrospective study of one thousand forty two breast cancer specimens was done from patients of different districts of Bangladesh during the period of January 2003 to April 2008. These specimens were received originally as surgical specimens fixed in $10 \%$ formalin at Anwara Diagnostic Center (ADC) in Dhaka. The specimens received were mastectomies, lumpectomies, Trucut biopsies, wide local excisions or chest wall skin biopsies. These surgical specimens were then fixed in $10 \%$ neutral buffered formalin for 24 hours and then the tissue was processed for routine hematoxylin and eosin staining through the steps of dehydration, clearing, paraffin impregnation and finally sectioning and staining.

Sections were cut at $4 \mu \mathrm{m}$ thickness, mounted onto salinized slides, and left to dry overnight at $37^{\circ} \mathrm{C}$. Sections were then deparaffinized and rehydrated. Antigen retrieval was achieved by heat retrieval using a bench autoclave. Briefly, slides were placed in Coplin jars containing enough TrisEDTA ( $\mathrm{pH} 9.0$ ) to cover the sections, then autoclaved at $121^{\circ} \mathrm{C}$ for 15 minutes for both ER and PR. For Her-2/neu, the slides were placed in Coplin jars containing enough Citrate buffer ( $\mathrm{pH} 6$, Dako, Denmark) to cover the sections, and autoclaved at $121^{\circ} \mathrm{C}$ for 10 minutes. After washing, the sections were covered by applying Endogenous Enzyme Block for 10 minutes (3\% hydrogen peroxide from Dako Denmark), the slides were then rinsed with Phosphate Buffer Solution (PBS). Slides were incubated with100$200 \mu$ l of primary antibodies for 30 minutes at room temperature in a moisture chamber, then rinsed in PBS. The dilution of the primary antibodies against ER (Dako clone 1D5, Denmark) and PR (Dako, clone PgR636, Denmark) was $1: 130$, and for Her-2/neu (cerb2oncoprotein, Dako, Denmark) was 1:50. Then the slides are incubated with Horse Redish Peroxidase (HRP) labelled polymer which is conjugated with secondary antibodies (Dako Label polymer). Finally, the sections were washed for four times in four minutes with changes of PBS, followed by adding 3,3 diaminobenzidine tetra hydrochloride (Dako) as a chromogen to produce the characteristic brown stain. The sections were then counterstained, dehydrated, and mounted for analysis. For each run of staining, a positive and negative control slide was also prepared. The positive control slides were prepared from breast carcinoma known to be positive for the antigen under study. The negative control slides were prepared from the same tissue block, but incubated with PBS instead of the primary antibody.

A semi-quantitative histochemical score was used to record results of ER and PR staining according to the system established by Allred et al. ${ }^{4}$. This system considers both the pro-portion and intensity of stained cells. The intensity score (IS) ranges from 0 to 3 , with 0 being no staining, 1 weak staining, 2 intermediate staining, and 3 intense staining. The proportion

score (PS) estimates the proportion of positive tumour cells and ranges from 0 to 5 , with 0 being non-reacting, 1 for $1 \%$ reacting tumour cells, 2 for $10 \%$, 3 for onethird, 4 for two-thirds , and 5 if $100 \%$ of tumour cells show reactivity. The PS and IS are added to obtain a total score (TS) that ranges from 0 to 8 . Tumour cells with a total score of 3 to 8 were considered positive, whereas those with TS less than 3 were considered negative cases.

Her-2/neu was scored on a 0 to 3 scale according to the criteria set by Dako. The staining was scored as: negative (0) when no membrane staining was observed, or when membranous staining was observed in less than $10 \%$ of the tumour cells; weak positive $(1+)$ if weak focal membrane staining was seen in more than $10 \%$ of the tumour cells; intermediate (2+) if weak to moderate, complete membrane staining was seen in more than $10 \%$ of the tumour cells; and strongly positive (3+) if intense membrane staining with weak to moderate cytoplasmic reactivity was seen in more than $10 \%$ of the tumour cells. The procedure was standardized in comparison with Dako Hercep kit.

\section{Results:}

The study population consisted of One thousand forty two female patients with invasive breast cancer with tumour tissues. Patients' mean age at diagnosis was 45.6 years. Infiltrating ductal carcinoma (IDC), not otherwise specified (NOS), accounted for 986 (94.6\%) of cases. Tweenty five patients were diagnosed with metastatic ductal carcinoma, 12 with infiltrating lobular carcinoma, nine with mucinous carcinoma, six with medullary carcinoma, two with metaplastic carcinoma, 
one with tubular carcinoma, and another one with Paget's disease ${ }^{5}$. Nine hundred eighty seven of the cases were graded histologically, 11 (1.1\%) were welldifferentiated (Grade I); 351 (35.6\%) were moderately differentiated (Grade II), and 625 (63.3\%) were poorly differentiated (Grade III) ${ }^{6}$.

\section{Table-I}

ER, PR, Her-2 Status of the histological specimens

\begin{tabular}{lcc}
$\begin{array}{l}\text { Histological } \\
\text { marker }\end{array}$ & No. & $(\%)$ \\
\hline HR (n=1042) & & \\
ER+ & 719 & $(69.0)$ \\
PR+ & 753 & $(72.3)$ \\
ER+,PR+ & 698 & $(67.0)$ \\
ER+,PR- & 21 & $(2.0)$ \\
ER-,PR+ & 55 & $(5.3)$ \\
ER-,PR- & 268 & $(25.7)$ \\
Her-2 (n=335) & & \\
Her-2 + & 95 & $(28.4)$ \\
\hline
\end{tabular}

HR, hormone receptor; ER, estrogen receptor; PR, progesterone receptor; Her-2, Human epidermal growth factor receptor 2; +, positive; -, negative

The estrogen and progesterone receptors and Her-2/neu expression status of the tumours is summarized in Table I. Of note, $69.0 \%(719 / 1042)$ of the specimens were ER positive, $72.3 \%$ (753/1042) were PR positive, and 28.4\% (95/335) of the specimens tested for Her-2/neu were positive. A tumour was considered to be "positive" or to over-express Her-2/neu if it had an IHC score of $3+$. There was a strong correlation seen between ER and PR status: when tumours were ER positive, 97.1\% (698/719) were also PR positive; similarly, when tumours were ER negative, 83.0\% (268/323) were simultaneously PR negative. Also, thirty tumours (9.0\%) were "triple negative" for ER, PR and Her-2 overexpression.

A consistent relationship was not seen between Her-2/ neu over-expression and HR status, likely because of a lack of statistical power. Overall, 22.7\% (10/44) of ER negative cases showed Her-2/neu over-expression compared to $29.2 \%$ (85/291) of the ER positive cases; however, this positive relationship was not statistically significant ( $\mathrm{p}=0.37$, chi-square). On the other hand, statistical power was gained when ER status was stratified by IHC score, and an inverse relationship was then seen between Her-2/neu over-expression and HR status (Table II). As the ER IHC scores increased from two to seven, Her-2/neu over-expression continually decreased from $63.6 \%$ to $0 \%$. In addition, when the data was divided into three groups based on low (0-2), medium (3-5) and high (6-8) ER IHC staining, statistical power was gained and a strong correlation was seen. Forty (40.3\%) percent of those specimens that had an IHC score of 0 to2 over-expressed Her-2/neu, 26.1\% of those with a score between 3 to 5 were her-2/neu positive, and only $22.0 \%$ of those with a score between 6 to 8 were Her-2/neu positive (correlation $r=-0.95$ ). This inverse correlation can also be seen by looking at the ER IHC score distribution of Her-2/neu + and Her2/neu - cases. A greater percentage of the Her-2+ cases are distributed amongst the lower ER IHC scores compared to the Her-2- cases, where a greater percentage of the Her-2- cases is distributed amongst the higher ER IHC scores. For example, 32.6\% of Her2/neu + cases had an ER IHC score of 0 to 2, and only $18.9 \%$ of the Her-2+ cases had a score between 6-8. Conversely, only 5.0\% of Her-2/neu - cases had an IHC score of two, while $26.7 \%$ of the Her-2- cases had an IHC score in the range of 6-8.

Biological tumour marker expression was not correlated with patient age at diagnosis (Table-III). Similarly, age was not significantly correlated with tumour grade or axillary lymph node status.

No significant associations were seen among tumour histological grade and dichotomous hormonal receptor or Her-2/neu expression status (Table-IV). Significant differences were seen however when the IHC scores of the histological grades were investigated. Table-V shows these trends. With greater histological differentiation, tumours had higher ER IHC scores. For example, a much higher percentage of Grade I tumours had ER IHC scores in the 5-8 range (72.7\%), meaning strongly positive, than did the Grade II (47.9\%) or Grade III tumours (40.8\%) ( $\mathrm{p}=0.015$, chi-square). The same could be seen with PR expression: $72.7 \%$ of Grade I, $52.7 \%$ of Grade II, and $45.9 \%$ of Grade III had IHC scores of 5-8 ( $p=0.035$, chi-square).

Cases with axillary lymph node metastases had a slightly higher Her-2/neu over-expression (29.5\%) (31/105) than those without (27.8\%) (64/230) ( $\mathrm{p}=0.75$, chi-square). 
Table-II

ER IHC scores in 335 Her-2-assessed cases

\begin{tabular}{|c|c|c|c|c|c|c|}
\hline \multirow{3}{*}{$\frac{\text { IHC Score }}{0}$} & \multicolumn{2}{|c|}{ Her-2+ [n=95] } & \multicolumn{2}{|c|}{ Her-2- $[\mathrm{n}=240]$} & \multirow{2}{*}{\multicolumn{2}{|c|}{ Her-2 Overexpression }} \\
\hline & No. & $(\%)$ & No. & $(\%)$ & & \\
\hline & 10 & $(10.5)$ & 34 & (14.2) & $10 / 44$ & $22.7 \%$ \\
\hline 2 & 21 & $(22.1)$ & 12 & $(5.0)$ & $21 / 33$ & $63.6 \%$ \\
\hline 3 & 3 & $(3.2)$ & 7 & $(2.9)$ & $3 / 10$ & $30.0 \%$ \\
\hline 4 & 20 & (21.1) & 54 & $(22.5)$ & $20 / 74$ & $27.0 \%$ \\
\hline 5 & 23 & $(24.2)$ & 69 & $(28.8)$ & 23/92 & $25.0 \%$ \\
\hline 6 & 8 & (8.4) & 38 & (15.8) & $8 / 46$ & $17.4 \%$ \\
\hline 7 & 0 & $(0.0)$ & 2 & $(0.8)$ & $0 / 2$ & $0 \%$ \\
\hline 8 & 10 & $(10.5)$ & 24 & $(10.0)$ & $10 / 34$ & $29.4 \%$ \\
\hline 0 (ER-) & 10 & $(10.5)$ & 34 & $(14.2)$ & $10 / 44$ & $22.7 \%$ \\
\hline 2-8 (ER+) & 85 & $(89.5)$ & 206 & $(85.8)$ & $85 / 291$ & $29.2 \%$ \\
\hline 2 & 21 & $(22.1)$ & 12 & $(5.0)$ & $21 / 33$ & $63.6 \%$ \\
\hline $3-5$ & 46 & $(48.4)$ & 130 & $(54.2)$ & $46 / 176$ & $26.1 \%$ \\
\hline $6-8$ & 18 & (18.9) & 64 & (26.7) & 18/82 & $22.0 \%$ \\
\hline
\end{tabular}

Table-III

Biological tumour marker expression by Age

\begin{tabular}{lccc} 
& $<50$ yrs old & 350 yrs old & p-value \\
\hline ER+ & $68.9 \%$ & $69.1 \%$ & 0.96 \\
PR+ & $71.2 \%$ & $74.1 \%$ & 0.32 \\
Her-2+ & $29.8 \%$ & $26.0 \%$ & 0.45 \\
\hline
\end{tabular}

There was a statistically significant difference in ER expression between the cases that had lymph node involvement (85.4\%) (205/240) and those that did not (64.1\%) (514/802) $(\mathrm{p}<0.001$, chi-square). A similar association was seen with PR expression: 86.7\% (208/ 240) of the cases with lymph node involvement were positive, while $68.0 \%$ (545/802) of the cases without lymph node involvement were $\mathrm{PR}+(\mathrm{p}<0.001$, chi-square $)$.
Table-IV

\begin{tabular}{|c|c|c|c|}
\hline \multicolumn{4}{|c|}{$\begin{array}{c}E R, P R, \text { and Her-2/neu expression by } \\
\text { Histological Grade }\end{array}$} \\
\hline Grade I (\%) & \multicolumn{2}{|c|}{ Grade II (\%) } & Grade III (\%) \\
\hline \multicolumn{4}{|l|}{ ER } \\
\hline Cases & 11 & 351 & 625 \\
\hline $\mathrm{ER}+$ & $9(81.8)$ & 241(68.7) & 429(68.6) \\
\hline \multicolumn{4}{|l|}{ PR } \\
\hline Cases & 11 & 351 & 625 \\
\hline $\mathrm{PR}+$ & $9(81.8)$ & $255(72.6)$ & 449(71.8) \\
\hline \multicolumn{4}{|l|}{ Her-2 } \\
\hline Cases & 5 & 106 & 210 \\
\hline Her+ & 0 & 34(32.1) & $58(27.6)$ \\
\hline
\end{tabular}


Table-V

\begin{tabular}{|c|c|c|c|c|c|c|c|c|}
\hline \multicolumn{9}{|c|}{ Histological grade by HR IHC scores } \\
\hline & \multicolumn{4}{|c|}{ ER } & \multicolumn{4}{|c|}{ PR } \\
\hline & Total & Grade 1 (\%) & Grade 2 (\%) & Grade 3 (\%) & Total & Grade $1(\%)$ & Grade 2 (\%) & Grade 3 (\%) \\
\hline 0 & 323 & $2(18.2)$ & $110(31.3)$ & 196 v(31.4) & 289 & $2(18.2)$ & $96(27.4)$ & $176(28.2)$ \\
\hline 2 & 64 & $0(0)$ & $17(4.8)$ & $42(6.7)$ & 63 & $0(0)$ & $17(4.8)$ & $41(6.6)$ \\
\hline 3 & 17 & $0(0)$ & $4(1.1)$ & $13(2.1)$ & 13 & $0(0)$ & $3(0.9)$ & $10(1.6)$ \\
\hline 4 & 181 & $1(9.1)$ & $52(14.8)$ & $119(19.0)$ & 170 & $1(9.1)$ & $50(14.2)$ & $111(17.8)$ \\
\hline 5 & 206 & 2 (18.2) & 74 (21.1) & $121(19.4)$ & 217 & 2 (18.2) & 72 (20.5) & 132 (21.1) \\
\hline 6 & 128 & 2 (18.2) & $52(14.8)$ & 68 (10.9) & 132 & $1(9.1)$ & $56(16.0)$ & 71 (11.4) \\
\hline 7 & 9 & $0(0)$ & $4(1.1)$ & $5(0.8)$ & 12 & $0(0)$ & $6(1.7)$ & $6(1.0)$ \\
\hline 8 & 114 & $4(36.4)$ & 38 (10.8) & $61(9.8)$ & 146 & 5 (45.5) & 51 (14.5) & 78 (12.5) \\
\hline $2-4$ & & 1 (9.1) & 73 (20.8) & $174(27.8)$ & & $1(9.1)$ & 70 (19.9) & 162 (25.9) \\
\hline $5-8$ & & $8(72.7)$ & 168 (47.9) & 255 (40.8) & & 8 (72.7) & 185 (52.7) & 287 (45.9) \\
\hline
\end{tabular}

\section{Discussion:}

The case series reported here is not population-based, but rather composed of referred cases for which hormonal receptors and Her-2/neu marker tests were requested by the physicians obtaining the tissues, and for which the patients were able to pay. Thus this is a selected case series. The degrees of difference in results from those obtainable from a population-based series are unknown. Most reports on hormonal receptor and Her-2/neu oncogene expression are similarly of selected case series, and on few occasions when population based series have been reported, interestingly, the frequencies of these markers have been remarkably similar to those found in the same geographic populations ${ }^{7}$.

In these contexts the most important results of the current study are those suggesting that the frequencies of ER, PR and Her-2/neu expression in tumours from Bangladeshi women with invasive breast cancer are very similar to those found in high income country population and most importantly, for ER and PR status, more than two third of all patients had tumours that were positive for ER, PR or both markers. While higher histological tumour grade was associated with lower hormonal receptor Allred scores, approximately $40 \%$ of grade III tumours were ER or PR moderately or strongly positive. Thus higher histological grade is a poor predictor of hormone receptor status.
The patients in this series are younger than those reported in most series from high income countries; it most likely reflects the sizes of the different age populations in Bangladesh from which these cases come. Younger patients are more likely to have higher grade tumours, as is observed in this series ${ }^{8}$. Younger age also appears to be an independent adverse prognostic risk factor ${ }^{8}$. The observation that cases with axillary nodal metastases were more frequently hormonal receptor positive, more likely reflects case selection than expression of biological natural history. Patients with hormonal receptor negative tumours have biologically and temporally more aggressive disease and, in the difficult economic and health system circumstances of most patients in Bangladesh, may be less likely to have tissue samples studied. Finally, in this study the previously noted inverse relationship between positive Her-2/neu over-expression and positive hormonal receptor status were also observed.

Diaz, Uy and other authors have emphasized the importance of tissue management prior to laboratory testing, which issues will be explored from this series in a future communication ${ }^{9,10}$. Here it is emphasized that efforts to place specimens in buffered fixatives within 30 minutes of surgical removal, and fixation for at least eight hours are critical factors in assuring likelihood of discovery of present hormonal receptor proteins, and preventing their destruction and non-detection. 
Because the presence of hormonal receptor proteins in tumours strongly predicts for response to hormonal therapies, which are less costly and toxic than systemic chemotherapies, the general observation that these proteins are present in more than 2/3rd of cases in Bangladeshi women, and that specific testing in individual cases can be successfully accomplished in Bangladesh are important issues for patients and their physicians ${ }^{2}$. The possibility of offering hormonal treatment to all patients with breast cancer in Bangladesh must now strongly be considered. Similarly, with respect to Her-2/neu testing, available data strongly suggest that only Her-2/neu positive tumour-bearing patients benefit from anthracycline chemotherapies, and thus this testing can potentially save two third of patients, for whom chemotherapy is under consideration, from the expense, gastro-intestinal, haematopoetic and cardiac toxicities of anthracycline treatments ${ }^{11,12,13}$.

In summary, frequency of hormonal receptor positive and Her-2/neu over-expressing tumours in Bangladeshi women with invasive breast cancer to be similar to those found in patients from high income countries.

\section{Limitation:}

Each breast specimen had taken different times to reach our laboratory as they had come from different parts of the country by different means. They were immersed in $10 \%$ formalin immediately after operation and kept in it while transportation. Then they were immediately re-fixed in $10 \%$ buffered neutral formalin (BNF) solution on receipt at our laboratory. So, in some cases, (where the specimens had travelled long ways from remote areas) complete tissue fixation may have occurred already when we received them, causing uneven fixation of the specimens, although the number is negligible. However, it may have minor influence on our testing accuracy.

\section{References:}

1. Olopade OI, Grushlo TA, Nanda R, Huo D. Advances in breast cancer: pathways to personalized medicine. Clin Cancer Res 2008; 14: 7988-7999.
2. Early Breast Cancer Trialists’ Collaborative Group. Effects of chemotherapy and hormonal therapy for early breast cancer on recurrence and 15 year survival: an overview of the randomized trials. Lancet 2005; 365: 1687-1717.

3. Pritchard KI. Endocrine therapy of advanced disease: analysis and implications of existing data. Clin Ca Res 2003; 9: 460S467S.

4. Allred DC, Harvey JM, Berardo M, Clark GM. Prognostic and predictive factors in breast cancer by immunohistochemical analysis. Mod Pathol 1998; 11: 155168.

5. Tavassoli FA, Devilee P, Editors: World Health Organization Classification of Tumours. Pathology and Genetics of Tumours of the Breast and Female Genital Organs. Lyon: IARC Press 2004.

6. Todd JH, Williams MR. Confirmation of a prognostic index in primary breast cancer. $\quad$ Br J Cancer 1987; 56:489-496.

7. Carey LA, Perou CM, Livasy CA et al. Race, breast cancer subtypes, and survival in the Carolina Breast Cancer Study. JAMA 2006; 295: 2492 - 2502.

8. Love RR, Duc NB, Dinh NV, Quy TT, Xin Y, Havighurst TC. Young age as an adverse prognostic factor in premenopausal women with operable breast cancer. Clinical Breast Cancer 2002; 2: 294-298.

9. Diaz LK, Sneige N. Estrogen receptor analysis for breast cancer: current issues and keys to increasing testing accuracy. Adv Anat pathol 2005; 12: 10-19.

10. Uy GB, Meis PM, Laudico AV et al. Immunohistochemistry assay of hormonal receptors in breast cancer: Philippines General Hospital Protocol and recommendations for improved testing. Philippine J Surgical Specialties 2007; 62: 128-134.

11. Paik S, Bryant J, Park C et al. C erb-2 and response to doxorubicin in patients with axillary lymph node-positive, hormone receptor-negative breast cancer. J Natl Cancer Inst 1998; 90: 1361-1370.

12. Paik S. Bryant J, Tan-Chiu E et al. Her-2 and choice of adjuvant chemotherapy for invasive breast cancer: National Surgical Adjuvant Breast and Bowel Project Protocol B-15. J Natl Cancer Inst 2000; 92: 1991-1998.

13. Pritchard KI, Shepherd LE, O’Malley FP et al. Her-2 and responsiveness of breast cancer to adjuvant chemotherapy. New Engl J Med 2006; 354: 2103-211. 